\title{
Messung von Lebensqualität im Kontext stationärer Pflege
}

\author{
Manuela Weidekamp-Maicher \\ (c) Der/die Autor(en) 2018 \\ K. Jacobs et al. (Hrsg.), Pflege-Report 2018 \\ https://doi.org/10.1007/978-3-662-56822-4_8
}

\section{Zusammenfassung}

Der Einzug des Lebensqualitätsbegriffs in das Pflegestärkungsgesetz entfachte in Deutschland eine neue Debatte über die Relevanz von Lebensqualität in der pflegerischen Versorgung. Der Beitrag erläutert den Begriff der Lebensqualität und gibt einen Einblick in ausgewählte Ansätze der Lebensqualität in der stationären Pflege. Anhand von Beispielen aus anderen Ländern zeigt er zudem verschiedene Möglichkeiten der Entwicklung und Implementierung neuer Instrumente, reflektiert allerdings auch die Chancen und Risiken der politischen Indienstnahme des Konzeptes.

The entry of the quality of life concept into the Care Support Act inspired a new debate in Germany about the relevance of quality of life in nursing care. The article explains the concept of quality of life and provides an overview of selected approaches to quality of life in nursing and residential care. Using examples from other countries, the author shows some possibilities for the development and implementation of new instruments and reflects the opportunities and risks of the political use of the concept.

\subsection{Theoretisches Verständnis von Lebensqualität in der stationären Pflege}

\subsubsection{Was ist Lebensqualität?}

Lebensqualität stellt einen komplexen Begriff dar, der in verschiedenen wissenschaftlichen Disziplinen und unterschiedlichen wissenschaftstheoretischen Kontexten verortet ist. Historisch betrachtet, zählt Lebensqualität zu den »neuen Wohlfahrtskonzepten " (Noll 2000), obwohl ihre politisch-normative Zielformel eines besseren, weil an der Qualität (anstatt der Quantität) orientierten, Lebens nicht neu ist. So entstanden bereits in der antiken Philosophie verschiedene Verständnisse eines "guten Lebens«, die teilweise Eingang in moderne Theorien gefunden haben. Die Popularität des Begriffs, die in der Medizin und Gesundheitsforschung in den 1940er
Jahren begann und in den Sozialwissenschaften ca. zehn Jahre später ihren Lauf nahm, gründete jedoch weniger auf der Neuentdeckung theoretischer Konzeptionen, sondern auf dem Gedanken der Messbarkeit des Konstruktes. Mit der (Neu-)Definition von Gesundheit ${ }^{1}$ sowie der zunehmenden Entwicklung sog. sozialer Indikatoren begann die »empirische Karriere« des Begriffs, deren Expansion in verschiedene Disziplinen reichte, u. a. in die Soziologie, Psychologie, Medizin, Ökonomie und Pflegewissenschaft. Dies führte zu einer steigenden Diversifikation des Konzeptes, sodass heute unterschiedliche Verständnisse von Lebensqualität vorliegen.

1 Die anlässlich der Gründung der Weltgesundheitsorganisation (im Jahr 1948) vorgelegte Definition betrachtet Gesundheit als "physical, mental, and social well-being and not merely the absence of disease or infirmity (www.who. int/about/mission/en). 
Widmet man sich der aktuellen Debatte um die Definition von Lebensqualität in der stationären Pflege, so gilt es zunächst zwischen der Qualität des Lebens und der Qualität der Pflege zu trennen und zugleich zu klären, von welchem Pflegebegriff die Rede ist. Aufgrund der starken Definitionsmacht des in der Pflegeversicherung verankerten Verständnisses von Pflege, das sich von dem breiter gefassten Begriff der Sorge (care) unterscheidet, wird unter dem Begriff der Pflegequalität i. d. R. die Güte jener Leistungen verstanden, die durch Pflege(fach-)kräfte erbracht werden. Dabei kommt der engere Begriff der Pflege zur Anwendung. Die Qualität des Lebens in einer Einrichtung hängt jedoch nicht nur von der Güte (fach-)pflegerischer Leistungen $\mathrm{ab}$, sondern wird von allen Merkmalen einer Einrichtung im ganzheitlichen Sinne tangiert. Je stärker die Abhängigkeit von (guter) Pflege und Versorgung und je verwobener das Leben bzw. Wohnen mit den Leistungen einer Einrichtung, umso stärker hängt die Lebensqualität der Bewohner und Bewohnerinnen von der Gesamtqualität eines Pflegeheims ab. Daher plädieren Zimmerman und Bowers (2001, S. 231) dafür, Pflege- und Lebensqualität nicht als distinkte, sondern als miteinander verwobene Konzepte zu betrachten.

Angesichts der starken Diversifikation in dem Feld stellt sich aktuell die Frage, welche wissenschaftliche Disziplin die theoretische Basis inkl. eines methodologisch-normativen Verständnisses von Lebensqualität in der stationären Pflege bestimmen soll. Während viele Autoren und Autorinnen hierfür die Pflegewissenschaft als verantwortlich sehen, plädieren andere für die Gerontologie. Wieder andere weisen auf die Rehabilitationswissenschaften hin (Bishop 2005) oder sehen die Definition von Lebensqualität aus einem gesamtgesellschaftlichen Verständnis von Pflege und ihren Aufgaben heraus begründbar (Vaarama und Pieper 2014). In Abhängigkeit von ihrer wissenschaftstheoretischen Herkunft lassen sich daher verschiedene Definitionen von Lebensqualität in der stationären Pflege unterscheiden. Auf der Grundlage einer umfassenden Konzeptanalyse pflegewissenschaftlicher Ansätze definiert Haas (1999b) Lebensqualität als "multidimensional evaluation of an individual's current life circumstances in the context of the culture and value systems in which they live and the values they hold «(S. 221). Aus der Sicht der Sozialen Gerontologie schlagen Higgs et al. (2003) einen Ansatz vor, in dem Lebensqualität anhand der Dimensionen: "control, autonomy, selfrealization and pleasure (S. 244) definiert wird. In der durch Lawton geprägten Tradition der psychologischen Gerontologie wird Lebensqualität wiederum als »the multi-dimensional evaluation, by both intrapersonal (subjective) and social-normative criteria, of the person-environment system " verstanden (Lawton 1991, S. 6). Aus rehabilitationswissenschaftlicher Sicht definiert Bishop (2005, S. 7) Lebensqualität als »the subjective and personally derived assessment of overall well-being that results from evaluation of satisfaction across an aggregate of personally or clinically important domains", wobei sie für die Langzeitpflege ein Modell bevorzugt, das die psychosoziale Anpassung an chronische Erkrankungen und Behinderungen in den Mittelpunkt stellt. Dem wachsenden Teil der Pflegeheimbewohner und -bewohnerinnen mit Demenz sollen wiederum zielgruppenspezifische Ansätze gerecht werden, wie z. B. das auf dem Capability-Ansatz beruhende Modell des Capability Index for Older People (ICECAP-0, Coast et al. 2008). Lebensqualität wird demnach durch Verwirklichungschancen in den Bereichen Verbundenheit, Sicherheit, Rolle, Freude sowie Unabhängigkeit definiert und fußt auf einem breiten Verständnis von Pflege als »social care (Makai 2012). Eine aus der gesellschaftlichen Positionierung von Pflege und Fürsorge abgeleitete Definition von Lebensqualität schlagen wiederum Vaarama und Pieper (2014) vor. »Pflegebezogene Lebensqualität" sollte demnach die Dimensionen emotionale Unterstützung bzw. Wohlbefinden, Servicequalität, Autonomie sowie soziale Unterstützung umfassen und durch einen gesamtgesellschaftlichen Konsens über Aufgaben und Verantwortlichkeiten in der Pflege legitimiert sein.

\subsubsection{Ansätze der Lebensqualität in der stationären Pflege}

Der Trend zur Pluralisierung bzw. Diversifikation zeigt sich nicht nur im Hinblick auf den Begriff der Lebensqualität an sich, sondern auch im Hinblick auf Ansätze der Lebensqualität in der stationären 
Pflege. Die meisten der aktuell vorliegenden Ansätze wurden in der Pflegewissenschaft und der Gerontologie entwickelt. In der Pflegewissenschaft (insbesondere in den USA) begann der Diskurs über Lebensqualität in den 1950er Jahren (Roop et al. 2012) und entwickelte sich rasch, sodass in den 1980er Jahren bereits eine Vielzahl unterschiedlicher Ansätze vorlag. Als Beispiele lassen sich das fünfdimensionale Modell von Padilla und Grant (1985), der für ältere Menschen entwickelte Ansatz von Foreman und Kleinpell (1990) sowie das vierdimensionale Modell von Ferrans (1990) nennen. Trotz der Tatsache, dass fast alle frühen pflegewissenschaftlichen Lebensqualitätsansätze der Onkologie entstammen, folgten sie keiner einheitlichen Forschungstradition. Nach der Darstellung von Haas (1999b, S. 216f) entwickelten sich in der Pflegewissenschaft vielmehr zwei "Denkrichtungen", von denen sich die erste am Ansatz der Lebenszufriedenheit, die zweite am Verständnis von Funktionsfähigkeit orientierte. Die zweitgenannte Strömung führte schließlich zur Adaption von Konzepten gesundheitsbezogener Lebensqualität, deren Einsatzbereich (in den 1990er Jahren) vor allem in der Bewertung medizinischer Interventionen gesehen wurde. Trotz begleitender Kritik am Begriff gesundheitsbezogener Lebensqualität, dem eine Reduktion auf negative Auswirkungen von Erkrankungen angelastet wurde (u. a. Cohen et al. 1996), fand dieser Eingang in empirische Forschung. Neuere theoretische Entwicklungen in diesem Feld finden sich in den Ansätzen von Ferrans et al. (2005) und Zubritsky et al. (2013). Während das erstgenannte Modell klinische Outcomes mit Aspekten der Lebensqualität kombiniert, bezieht sich das zweitgenannte auf Menschen mit kognitiven Einschränkungen und definiert Lebensqualität aus deren Rolle als Empfänger bzw. Empfängerinnen pflegerischer Leistungen.

Betrachtet man neuere Entwicklungen, so zeichnet sich aktuell ein Bemühen um stärkere Abgrenzung zwischen Lebensqualität und Gesundheit bzw. weiteren Begriffen ab, verbunden mit dem Ziel der Klärung bzw. gar (Neu-)Positionierung des Lebensqualitätskonzeptes in der Pflege. So unternehmen Plummer und Molzahn (2009) eine kritische Konzeptanalyse aktueller pflegewissenschaftlicher Theorien, um genuine Merkmale eines pflegewissen- schaftlichen Verständnisses von Lebensqualität zu bestimmen. Die zu diesem Zweck herangezogenen Theorien definieren Lebensqualität als subjektive, dynamische, d. h. sich verändernde Kategorie. Trotz eines fehlenden Konsensus über die Zahl und inhaltliche Spezifikation relevanter Dimensionen betrachten die Autorinnen Lebensqualität als ein zentrales »Metaparadigma« der Pflegetheorie und - praxis und plädieren dafür, das Metaparadigma Gesundheit durch jenes der Lebensqualität zu ersetzen.

Definitorische Abgrenzungsnotwendigkeiten bestehen ebenfalls zwischen dem Begriff der Lebensqualität und den Begriffen Wohlbefinden und Wohlergehen. So wird Wohlergehen (comfort) in der Internationalen Klassifikation für die Pflegepraxis (ICNP) mithilfe des Begriffs Wohlbefinden (wellbeing) definiert (International Council of Nurses 2017, S. 184) ${ }^{2}$. Wohlbefinden umfasst wiederum psychologische, spirituelle oder psychosoziale - selten auch körperliche - Aspekte und gilt als Bestandteil von Lebensqualität bzw. wird mit dieser häufig gleichgesetzt (vgl. Kelley-Gillespie 2009). Auf der Grundlage von 18 Konzeptanalysen schlagen daher Pinto et al. (2017) vor, zwischen Wohlergehen bzw. Wohlbefinden zu trennen und sie als distinkte, mit Lebensqualität nicht gleichsetzbare Begriffe zu verwenden.

Nach einer für ältere Menschen geeigneten Definition von Lebensqualität, die zugleich als Outcome der (Alten-)Pflege herangezogen werden kann, fragt schließlich auch Boggatz (2016). Im Rahmen einer Konzeptanalyse unterscheidet der Autor drei zentrale inhaltliche Bedeutungen des Begriffs: a) Lebensqualität als Konstellation »zufriedenstellender « und für ein "gutes Altern« geeigneter Lebensbedingungen, b) Lebensqualität als subjektives Wohlbefinden, das sowohl kognitive (Lebenszufriedenheit) als auch emotionale (emotionales Gleichgewicht) Aspekte umfasst, sowie c) Lebensqualität als subjektiv wahrgenommene Verwirklichung zentraler Entwicklungsziele bzw. -potenziale in relevanten Dimensionen des menschlichen Daseins. Der Autor (ebenda, S. 66f) geht davon aus, dass die letztgenannte Bedeutung für die Pflege hochbetagter Menschen am besten geeignet

2 Vgl. auch Klassifikation der Pflegediagnosen (Herdman und Kamitsuru 2017). 
sei. Eine Theorie, die sich einer derartigen Definition bedient, ist z. B. der Ansatz des psychologischen Wohlbefindens von Ryff und Keyes (1995). Trotz des Vorliegens entsprechender Messinstrumente bemängelt Boggatz (ebenda) jedoch deren Eignung für die Pflege. Relevanter scheint jedoch das Fehlen einer allgemein akzeptierten Theorie der Lebensqualität in der stationären Pflege zu sein, die über empirisch ermittelte und statistisch entwickelte Modelle hinausgeht. So kommen selbst theoretische Analysen zu dem Schluss, dass Lebensqualität in der Pflege(-wissenschaft) ein wenig greifbares, abstraktes, relatives, grenzenloses und mit einer sich verändernden Zielsetzung verbundenes Konzept ist, das sowohl strukturell als auch mit Blick auf die Abgrenzung zur Gesundheit nicht klar fokussiert werden kann (Plummer und Molzahn 2009). Zudem zeichnet sich nach wie vor eine Zunahme operationaler Definitionen von Lebensqualität $\mathrm{ab}$, die je nach Vorhandensein bzw. Art der theoretischen Fundierung unterschiedliche Dimensionen postulieren und einer differenten Strukturlogik folgen. Einige aktuelle Arbeiten versuchen daher eine Synthese verschiedener Ansätze zu realisieren (Halvorsrud und Kalfoss 2007, Kelley-Gillespie 2012). Bei näherer Betrachtung zeigt sich jedoch, dass deren Ergebnisse für die Erfassung von Lebensqualität in der stationären Pflege nicht geeignet sind, so dass es relevanter wäre, Lebensqualität (aus Bewohner- und Bewohnerinnensicht) zunächst auf theoretischem Wege zu bestimmen, bevor eine Generierung weiterer Ansätze auf der Grundlage bestehender Instrumente erfolgt.

Zusammenfassend lässt sich festhalten, dass Lebensqualität einen in der Wissenschaft verbreiteten, jedoch komplexen und in Abhängigkeit von seiner theoretischen Vorortung unterschiedlich definierten Begriff darstellt. Sowohl die Pflegewissenschaft als auch die Gerontologie haben verschiedene Definitionen und Konzepte vorgelegt, verfügen allerdings über keine konsensuell anerkannte Theorie, die Lebensqualität aus Sicht von Bewohner und Bewohnerinnen stationärer Einrichtungen allumfassend definiert und die zugleich als Grundlage einer vergleichenden Messung herangezogen werden könnte.

\subsection{Messung von Lebensqualität in der stationären Pflege - ausgewählte Instrumente im internationalen Vergleich}

\subsubsection{Gesellschaftspolitische Hinter- gründe nationalspezifischer Bemühungen um Messung von Lebensqualität in der stationären Pflege}

Die Diskussion um Messung von Lebensqualität in der Pflege hat sowohl wissenschaftlich-theoretische als auch gesellschaftlich-politische Hintergründe. Letztere sind im internationalen Vergleich unterschiedlich beschaffen, auch wenn sie alle Ausdruck aktueller Bemühungen um die Verbesserung der Qualität in der Pflege sind. Während ähnliche Vorstöße bisher jedoch vornehmlich den Interessen der Einrichtungen dienten, setzte sich in den letzten Jahren sowohl in den europäischen Ländern als auch den USA die Erwartung durch, die Weiterentwicklung von Qualität unter Einbeziehung der Sicht von Bewohnern und Bewohnerinnen und ihrer Lebensqualität $\mathrm{zu}$ forcieren. Diese Entwicklung trägt Züge eines veränderten paradigmatischen Verständnisses von Qualität in der Pflege, bei dem ein Person-zentrierter Ansatz als wichtigster Treiber eines als notwendig erachteten Kulturwandels betrachtet wird.

In den USA bildet Lebensqualität einen zentralen Begriff einer nationalen Kampagne, die als »nursing home culture change movement « ${ }^{3}$ bezeichnet wird und der sich Akteure aus Politik, Wissenschaft und gesundheitlich-pflegerischer Versorgung angeschlossen haben. Wichtige Vorreiter der Bewegung sind Robert L. Kane und Rosalie A. Kane, die zur Entwicklung von Instrumenten zur Messung von Lebensqualität in der stationären Pflege maßgeblich beigetragen haben (Kane und Kane 2015). Zentral für den beabsichtigten Kulturwandel ist die Abkehr von einer am Prinzip der Sicherheit orientierten Pflege, hin zu einem individualisierten Verständnis pflegerischer Versorgung (Love 2011). Eine solche Ausrichtung wird nicht nur als Schlüssel zur Verbesserung der Lebensqualität von Pflegeheimbe- 
wohner*innen betrachtet, sondern als zentraler Mechanismus zur Verbesserung der Qualität von Pflege insgesamt (Zimmerman et al. 2014; Kane 2015). Durch den Perspektivwechsel werden Pflegeheimbewohner und -bewohnerinnen zu zentralen Stakeholdern, da sie sowohl in die Definition (=patient-defined outcomes) als auch die Bewertung (= patient-related outcomes) der Lebensqualität einbezogen werden sollen. ${ }^{4}$

\section{- Bemühungen um den Wandel}

von einem Institution-zentrierten zu einem Person-zentrierten Qualitätsverständnis lassen sich ebenfalls in Europäischen Ländern beobachten. So schreiben Colombo und Murakami: »Traditionally, approaches to measuring the quality of long-term care have centred on measuring inputs such as staff to care-recipient rations, but recognition that outcomes make a better basis for measurement has grown over time. More recently, pressure has grown to embrace quality of life and person-centredness as the appropriate focus of measurement « (Colombo und Murakami 2013, S. 16). (...) Quality of life, which is often identified as a main dimension of LTC quality, can be regarded as the overarching goal of LTC quality initiatives « (ebenda, S. 17). Die Realisierung eines solchen Vorhabens erweist sich jedoch als ein komplexes Unterfangen. Dennoch haben sich einige Länder auf den Weg gemacht und sowohl entsprechende Instrumente als auch Systeme zur öffentlichen Berichterstattung realisiert. Die gewonnenen Daten basieren in der Regel auf standardisierten Befragungen von Pflegeheimbewohnern und -bewohnerinnen oder ihren gesetzlichen Vertretern und beziehen auch sog. »intangible indicators" wie Zufriedenheit oder Wohlbefinden ein. Im Rahmen des nachfolgenden Unterkapitels werden ausgewählte Instrumente im Lichte konstituierender gesellschaftlich-politischer Bedingungen aus drei verschiedenen Ländern vorgestellt.

4 Durch den im Jahr 2017 erfolgten Regierungswechsel und die damit verbundenen Änderungen der gesetzlichen Lage ist die Umsetzung der Kampagnenziele jedoch ungewiss.

\subsubsection{Ausgewählte Messinstrumente}

\section{- Deutschland}

Die Debatte über Messung von Lebensqualität in der Pflege steht in Deutschland maßgeblich im Zusammenhang mit den Änderungen der Pflegeversicherung. Durch den Einzug des Lebensqualitätsbegriffs in das Pflegestärkungsgesetz $\mathrm{II}^{5}$ kam es zu einer wissenschaftlichen Diskussion um geeignete Definitionen und Erfassungsmethoden. Das im Kontext der pflegerischen Versorgung verhandelte Verständnis von Lebensqualität verdankt seine Aktualität daher der politisch motivierten Herausforderung, Schnittstellen zwischen Pflege- und Lebensqualität zu bestimmten und letztere im Kontext der Pflege zu definieren und zu erfassen. Trotz dessen liegt bisher kein landesweiter Ansatz zur Messung von Lebensqualität vor. In den vergangenen Jahren entstanden jedoch einige Instrumente, die im Folgenden vorgestellt werden sollen.

Eines der bisher entwickelten Instrumente bildet das Befragungsinstrument QUISTA (=Messung von Lebensqualität in der stationären Pflege, Schenk et al. 2013). Lebensqualität wird hier als der Grad der Übereinstimmung zwischen individuellen Ansprüchen der Bewohner und Bewohnerinnen und deren subjektiv wahrgenommener Erfüllung definiert. Die insgesamt zehn Dimensionen des Instruments (dazu gehören: 1) etwas Sinnvolles bzw. Freude Stiftendes tun, 2) sich mit anderen austauschen, 3) Selbstbestimmung und Selbstständigkeit, 4) Intimsphäre, 5) Ruhe und Privatsphäre, 6) Abwechslung, Beschäftigung und Aktivität, 7) sich heimisch fühlen, 8) sich sicher und behütet fühlen, 9) Gesundheit, 10) im Dialog stehen und Informationen erhalten) wurden auf der Grundlage qualitativer Befragungen von Pflegeheimbewohnern und -bewohnerinnen generiert. Trotz fehlender Trennschärfe zeigen sie exemplarisch, dass Pflegeheimbewohner und -bewohnerinnen ihre Lebensqualität unterschiedlich definieren: zum einen anhand bestimmter Werte, wie z. B. Selbstbestimmung und Sicherheit, zum anderen anhand relevanter Lebensbereiche, z. B. Aktivitäten und soziale Kontakte.

5 Vgl. § 113b, der die Aufgaben des neu geschaffenen Qualitätsausschusses definiert (Abs. 10.4 Pt. 4). 
Ein ähnlich konzipiertes Instrument, das Instrument zur Praxisnahen Erfassung von Lebensqualität (INSEL), entstand im Rahmen eines gleichnamigen Projektes (Oswald et al. 2014). Auch dieses orientiert sich an mehrdimensionalen Vorstellungen von Lebensqualität, wobei sich einige Dimensionen an bereits bestehende Ansätze (Kane et al. 2003) anlehnen. Das Instrument kombiniert quantitative und qualitative Befragungsergebnisse und verbindet die Perspektive der Bewohner und Bewohnerinnen mit der Perspektive des Pflegepersonals. Lebensqualität wird anhand von zwölf Dimensionen definiert, die dazu dienen, Person-zentrierte Aspekte durch offene Fragen zu erfassen. Können sich Bewohner und Bewohnerinnen nicht äußern, werden Angehörige stellvertretend befragt. Ähnlich wie das Instrument QUISTA kann INSEL verschiedene Funktionen erfüllen: Im Längsschnitt angewandt, dient es der Unterstützung des internen Qualitätsmanagements, der Suche nach bewohnerzentrierten Entscheidungen sowie dem Monitoring von Lebensqualität.

Angesichts der Forderung nach mehr Validität im Sinne von "patient-defined outcomes" entstanden in den vergangenen Jahren Instrumente, die dem individuell-subjektiven Charakter von Lebensqualität in besonderer Weise gerecht werden sollen. Sie begegnen der Erfassung von Lebensqualität anhand persönlich wichtiger Themen der Bewohner und Bewohnerinnen und vermeiden es, Lebensqualität anhand interpersonell vergleichbarer Dimensionen zu erfassen. Ein solches Verständnis basiert auf sog. idiographischen Modell von Lebensqualität ${ }^{6}$, das davon ausgeht, dass Menschen einmalige und nicht miteinander vergleichbare Aspekte der Lebensqualität als bedeutsam erachten. Das Ergebnis solcher Erhebungen sind Zusammenstellungen von Kennzeichen der Lebensqualität, die für Bewohner und Bewohnerinnen in ihrem Verständnis als individuelle Sub-

6 Vgl. dazu das von McGee et al. (1991) entwickelte Instrument SEIQoL (= The Schedule for the Evaluation of Individual Quality of Life), das als erstes dieser Art entstand. Im entgegengesetzten Verständnis, d. h. sog. nomothetischen Modellen der Lebensqualität, kommt es im Rahmen der Instrumentenentwicklung zu einer standardisierten Dimensionsbildung, auf deren Basis Vergleiche zwischen Personen und Einrichtungen möglich sind. jekte relevant sind. In Einrichtungen können sie zur Gestaltung des Alltags und zur Unterstützung Bewohner-zentrierter Entscheidungen dienen. Ein Instrument, das diesem Modell entspricht, ist der LQ-Index von Herold-Majumdar und Behrens (2012).

Neben den genannten Instrumenten gibt es in Deutschland erstmals einen Ansatz zur Entwicklung von Indikatoren der Ergebnisqualität, darunter auch Indikatoren der Lebensqualität, die vor allem über Befragungen von Bewohnern und Bewohnerinnen und Angehörigen erhoben werden. Die Entwickler (Wingenfeld et al. 2011) haben zudem erste Vorschläge zur Risikoadjustierung vorgelegt. Indikatoren, die der Erfassung von Lebensqualität dienen, beziehen sich auf Erfahrungen der Bewohner und Bewohnerinnen (=Pflegequalität aus Nutzer- und Nutzerinnensicht), beruhen jedoch nicht auf theoretischen Definitionen von Lebensqualität, obwohl diese in die Vorüberlegungen eingeflossen sind (u. a. Glatzer und Zapf 1984). Die Operationalisierung orientiert sich am Ansatz von Pringle und Doran (2003, zit. in Wingenfeld et al. 2011, S. 16), in dem es um die Konzipierung sog. "perceptual outcomes" geht. In einer nachfolgenden Studie (mit 62 Pflegeheimen und über 3.000 Pflegebedürftigen in fünf Bundesländern) wurden insgesamt 15 der von Wingenfeld et al. (2011) entwickelten Indikatoren hinsichtlich ihrer bundesweiten Verwendbarkeit untersucht (Görres et al. 2017). Die Ergebnisse deuten auf die prinzipielle Eignung einiger Indikatoren für den breiten Einsatz hin; deren Zusammenstellung zeigt aber, dass sich darin ein auf Funktionsfähigkeit fokussiertes Verständnis von Lebensqualität spiegelt und Aspekte, die aus Bewohner- und Bewohnerinnensicht von besonderer Relevanz sind, nicht vorkommen.

\section{- Großbritannien}

Eine landesspezifische Debatte um die Notwendigkeit eines Kulturwandels - vor allem in der stationären Pflege - verbunden mit dem Ruf nach einer evidenzbasierten Politik und Praxis lässt sich auch in Großbritannien beobachten (Meyer und Owen 2008). Sichtbar wurden diese Bemühungen u. a. durch das Programm »My Home Life«, dem sich verschiedene Organisationen, u. a. die »Quality of Care for Older People Research Group«, ange- 
schlossen haben (u. a. NCHRDF 2006). Die als mangelhaft kritisierte Pflege und die fehlende Transparenz der Qualität von Pflegeeinrichtungen wurden in den 2000er Jahren öffentlich stark kritisiert mit dem Ergebnis, dass eine Vielzahl sog. "care rating websites « entstand. Um die verschiedenen Informationen zur Qualität von Pflege zu bündeln, richtete die Regierung ein Portal ein, in dem sich heute auch Informationen zur Lebensqualität in der stationären Pflege befinden. ${ }^{7}$

Seit 2011 finden in Großbritannien systematische Erhebungen von Indikatoren der Lebensqualität - sowohl in der stationären als auch der ambulanten Pflege - statt. Das zentrale Instrument zur standardisierten Erfassung von Lebensqualität bei Empfängern und Empfängerinnen sozialer Dienstleistungen ist das Adult Social Care Outcomes Toolkit (ASCOT), eine Sammlung mehrerer Teilinstrumente, die für verschiedene Arten sozialer Dienstleistungen, Versorgungssettings (ambulant und stationär) und Zielgruppen Erwachsener dienen (Netten et al. 2010, 2011; Forder et al. 2007). Ihre Zielsetzung besteht darin, vergleichbare Ergebnisse zur Lebensqualität auf kommunaler bzw. regionaler Ebene zur Verfügung zu stellen. Das Instrument liegt in verschiedenen Versionen (zum individuellen Ausfüllen, als Beobachtungsversion) sowie in einfacher Sprache vor (Turnpenny et al. 2015). Auch eine Proxy-Version wurde kürzlich entwickelt, mit der andere Personen im Namen der Nutzer und Nutzerinnen sozialer Dienstleistungen antworten können (Rand et al. 2017).

ASCOT ist darüber hinaus Baustein des Adult Social Care Outcomes Framework (ASCOF), einer umfassenden Sammlung von Outcomes, die der Berichterstattung zur (Lebens-)Qualität durch soziale Dienstleistungen dient (Department of Health 2014). Die Befragungen zur Lebensqualität sind zudem eingebettet in den Adult Social Care Survey (ASCS) mit jährlichen Erhebungen, dessen Ziel darin besteht, Nutzer- und Nutzerinnenerfahrungen zu erfassen und Referenzwerte zu bilden (Department of Health 2017). Im Rahmen des o. g. Survey wird zudem eine Reihe weiterer Variablen erfasst,

7 Weitere Informationen unter: www.nhs.uk/Conditions/ social-care-and-support-guide/Pages/what-is-social-care. aspx die in ihrer Funktion als Moderatoren untersucht werden. Beispiele sind der Schweregrad der Erkrankung, die ökonomische Situation Betroffener, deren Alter und soziale Ressourcen. Ergänzt werden die Ergebnisse durch Befragungen informeller Pflegekräfte, den Survey of Adult Carers in England (SACE), der in England im Jahr 2016/2017 zum dritten Mal durchgeführt wurde (Forder et al. 2016).

Zentral für die Messung von Lebensqualität ist der Begriff der Social Care-related Quality of Life (SCRQol). Er basiert auf wohlfahrtsökonomischen Ansätzen und spiegelt jenen Zuwachs an Wohlbefinden (»capacity for benefit») wider, den Befragte in Relation zur Referenzgruppe ohne die in Frage stehende Dienstleistung erleben (Forder et al. 2007, S. 19). Lebensqualität wird anhand von acht Dimensionen definiert: "personal cleanliness and comfort «, »safety«, »control over daily life «, »accommodation cleanliness and comfort «, »food and nutrition«, »occupation «, »social participation and involvement« und »dignity« (Netten et al. 2010, S. 7). Die Konstruktvalidität des Instruments konnte auch bei älteren Menschen - weitgehend bestätigt werden (Malley et al.2012), wobei die Ergebnisse immer wieder zeigen, dass basale Bedürfnisse besser erfüllt werden als höhere Bedürfnisse. Die SCT-4-Version des ASCOT besteht aus insgesamt neun Fragen, wobei zu jeder Dimension eine Frage gestellt wird - mit Ausnahme der letzten Dimension - »dignity « - zu der zwei Fragen vorliegen. Der zu errechnende Index-Score (sowohl Gesamt- als auch dimensionsspezifischer Score) stellt ein gewichtetes Maß dar, wobei die Gewichte auf Grundlage der o. g. Befragungen ermittelt werden.

Die vierstufige Version des ASCOT wurde inzwischen ins Niederländische übersetzt. Eine erste Pilotierung zeigt, dass trotz zufriedenstellender Reliabilität und Validität sowohl Ähnlichkeiten als auch Differenzen im Ländervergleich bestehen (van Leeuwen et al. 2015). Wesentliche Unterschiede zeigten sich bei den Items zu Würde (»dignity«). Darüber hinaus wird im Rahmen eines weiteren Vorhabens mit dem Titel Exploring Comparative Effectiveness and Efficiency in Long-term Care (EXCELC) eine Übersetzung ins Finnische und Deutsche vorgenommen. Im weiteren Schritt soll der Einfluss der Betreuungs- und Pflegeleistungen auf die Lebensqualität der Klienten und Klientinnen 
und ihrer Angehörigen ländervergleichend untersucht werden.

\section{- Niederlande}

Auch in den Niederlanden sind seit einigen Jahren Bemühungen um die Entwicklung einer nationalen Sammlung von Indikatoren zur Messung von Pflege- und Lebensqualität inkl. entsprechender Erhebungsinstrumente sichtbar. Zwecks einer landesweiten Erfassung von Pflege- und Lebensqualität und ihrer öffentlichen Berichterstattung wurden im Jahr 2007 das Rahmenmodell Quality Framework Responsible Care (Steering Committee Responsible Care 2007) sowie der Consumer Quality Index (CQ-index ${ }^{\circledR}$ ) Long-Term Care, ein Instrument bzw. Kriterienkatalog zur Messung von Pflege- und Lebensqualität in der häuslichen und stationären Pflege, entwickelt. Im Unterschied zu Großbritannien, wo die Steuerung des Entwicklungsprozesses auf Regierungsebene angesiedelt war, entstand das niederländische Rahmenmodell inkl. des dazugehörigen Erhebungsinstruments im Rahmen eines konsensorientierten Verfahrens, an dem alle für die Pflege zuständigen Akteure beteiligt waren (Delnoij et al. 2010). Eine zentrale Rolle im Entwicklungsprozess spielte das Centrum Klantervaring Zorg (= Dutch Centre for Consumer Experience in Health Care), eine Stiftung, die u. a. aus Mitteln des niederländischen Gesundheitsministeriums gefördert wird und die für die Koordination der Datenerhebung zuständig ist. Der Consumer Quality Index, der auch für andere Versorgungskontexte zur Verfügung steht, ist eine eingetragene Marke der Stiftung. Seit der Einführung des neuen Instruments im Jahr 2007 erfassen alle Pflegeeinrichtungen je zwei Jahre die Qualität ihrer Pflege inkl. ausgewählter Aspekte der Lebensqualität. Eine öffentliche Berichterstattung wird auf den Webseiten www.kiesbeter.nl, einer Seite des National Health Care Institute, sowie www.jaarverslagenzorg.nl, einer Seite des niederländischen Gesundheitsministeriums, vorgenommen.

Der Consumer Quality Index lehnt sich an zwei bereits existierende Instrumente an: das Consumer Assessment of Healthcare Providers and Systems ${ }^{\circledR}$ (CAHPS) der US-amerikanischen Agency for Healthcare Research and Quality (AHRQ), sowie ein Instrument mit dem Namen Quality Of Care Through the Patients' Eyes (QUOTE), eine Entwicklung des Netherlands Institute for Health Services Research (NIVEL). Das zweitgenannte Instrument liegt in verschiedenen Versionen vor, u. a. zur Erfassung der Qualität pflegerischer Versorgung aus Sicht älterer Pflegebedürftiger in der Kommune (Sixma et al. 2000). Das theoretisch-konzeptionelle Verständnis von Lebensqualität basiert auf Erfahrungen der Bewohner und Bewohnerinnen (= patient experience indicators) und stellt die wahrgenommene Pflegequalität in den Mittelpunkt der Betrachtung. Der CQ-index ${ }^{\circledR}$ Long-Term Care verbindet daher verschiedene Arten von Outcomes: klinische Daten aus der Pflegedokumentation, Indikatoren der Pflegequalität sowie subjektive Bewertungen der Bewohner und Bewohnerinnen. Die zusätzlich erhobenen Angaben zur Relevanz verschiedener Versorgungsmerkmale machen es möglich, die Wichtigkeit bestimmter Leistungen aus Nutzer- und Nutzerinnensicht einzuschätzen. Neben einem standardisierten Fragebogen für die mündliche Bewohnerbefragung liegen ein schriftlicher Fragebogen für rechtliche Betreuer und Betreuerinnen von Menschen mit Demenz sowie ein schriftlicher Fragebogen für Empfänger ambulanter Pflegeleistungen vor. Der für Pflegeheimbewohner und -bewohnerinnen entwickelter Bogen ist deutlich länger als die in Großbritannien entwickelte Version, sodass die Dauer eines Interviews im Durchschnitt bei 43 Min. liegt (Winters et al. 2010, S. 4).

Die aktuelle Version des CQ-Index ${ }^{\circledR}$ Long-Term Care umfasst zehn (Qualitäts-)Bereiche: 1) Care/ treatment/life plan, 2) Communication \& information, 3) Physical well-being, 4) Safety care content, 5) Domestic \& living conditions, 6) Participation \& social handiness, 7) Mental well-being, 8) Safety living/residence, 9) Sufficient and competent staff, 10 ) Coherence in care (Steering Committee Responsible Care 2007, S. 10). Aspekte der Lebensqualität beziehen sich auf die Bereiche physisches bzw. körperliches Wohlbefinden bzw. Gesundheit, Wohnen und Lebensbedingungen, Partizipation und soziales Wohlergehen sowie mentales Wohlbefinden (Triemstra et al. 2010). Die risikoadjustierten Indikatoren haben einen relativen Charakter, sodass Einrichtungen die Möglichkeit haben, ihre eigene Qualität mit den Ergebnissen anderer Einrichtungen zu vergleichen (Damman et al. 2009). 
Bisherige Erfahrungen zeigen, dass sich die meisten Indikatorenwerte im Zeitverlauf verbessert haben und dass Verschlechterungen im Vergleich zu Verbesserungen durchgehend geringer ausfallen. Die höchsten Verbesserungen ergaben sich bei jenen Indikatoren, die auf Bewohner- und Bewohnerinnenbefragungen zurückgehen. Bei den Qualitätsbereichen waren die größten Zuwächse im Bereich der Pflegeorganisation zu verzeichnen, vor allem in den Bereichen Pflegeplanung (»care plan«), Entscheidungen gemeinsam treffen (»shared desicion making «) sowie Kohärenz in der Pflege (»coherence in care «) (Zuidgeest et al. 2012, S. 7). Auf Einrichtungsebene zeigte sich, dass sich im Zeitverlauf vor allem jene Einrichtungen besserten, die in ihren Indikatorenwerten bereits recht hoch lagen. Als ein wesentlicher Grund dafür wird die öffentliche und transparente Darstellung der Ergebnisse vermutet. $\mathrm{Ob}$ die beobachtbaren Änderungen tatsächliche Verbesserungen der Pflege- und Lebensqualität spiegeln, muss durch langfristige Analysen überprüft werden. Forschung weist jedenfalls darauf hin, dass positive Effekte einer transparenten Berichterstattung erst dann wirksam werden können, wenn die Qualitätsinformation entsprechend der Intention der Entwickler interpretiert wird und sie Eingang in konkrete Entscheidungen der Pflegebedürftigen findet. Daher plädieren viele dafür, die Erforschung der Interpretationsmuster sowie der Bedeutung dieser Art von Gesundheitskommunikation für Nutzer und Nutzerinnen pflegerischer Leistungen zur Grundlage der Weiterentwicklung der Instrumente $\mathrm{zu}$ machen (Damman 2010, S. 163). Offen ist zudem die Frage, wer von dieser Art der Qualitätsdarstellung profitiert und ob vergleichende Lebensqualitätsdaten einen Beitrag zum Empowerment von Menschen mit Pflegebedarf leisten.

Resümierend kann festgehalten werden, dass die Weiterentwicklung der Qualität in der Pflege, insbesondere im Hinblick auf den stationären Kontext - auch international - zu einer wichtigen gesamtgesellschaftlichen Aufgabe geworden ist. Neu an der aktuellen Diskussion ist allerdings, dass Verbesserungen der Pflegequalität durch eine Lebensqualitätsorientierung forciert werden sollen, was eine explizite Einbeziehung der Bewohner und Bewohnerinnen voraussetzt. Was konkret unter Le- bensqualität verstanden werden soll und wie das Konzept Eingang in die pflegerische Versorgung findet, ist Gegenstand von Debatten zwischen beteiligten Akteuren. Bei der Entwicklung konzeptioneller Grundlagen zur Erfassung von Lebensqualität inkl. der dazugehörigen Instrumente sind verschiedene Länder unterschiedliche Wege gegangen. Einige davon, z. B. Deutschland, befinden sich noch mitten im Konzeptionsprozess. Über die Wirkungen der nationalspezifischen »Projekte«, z. B. ob sie zu realen Verbesserungen der Lebensqualität geführt oder lediglich die Erwartungen an die stationäre Pflege erhöht haben, ist aufgrund knapper Laufzeiten wenig bekannt. Dies dürfte jedoch eine zentrale Forschungsfrage der Zukunft werden.

\subsection{Fazit und Diskussion}

Zusammenfassend zeigt sich, dass eine Vielzahl unterschiedlicher Definitionen von Lebensqualität existiert. Sowohl die Pflegewissenschaft als auch die Gerontologie blicken auf eine lange Tradition der Lebensqualitätsforschung zurück, verfügen jedoch über keine konsensorientierte Theorie, die Lebensqualität aus Sicht von Bewohner und Bewohnerinnen stationärer Einrichtungen definiert. Erschwert wird die Bildung einer Theorie dadurch, dass deren Form durch die geplante Verwendung inkl. der sie bestimmenden politischen Rahmenbedingungen a priori geprägt wird. Die aktuelle Diskussion über den Begriff der Lebensqualität in der stationären Pflege sowie die Beispiele zur Messung von Lebensqualität inkl. ihrer versorgungspolitischen Verwertung machen in diesem Zusammenhang auf bestimmte Dilemmata aufmerksam. Eines dieser Dilemmata liegt bereits in der Logik begründet, durch die der Lebensqualitätsbegriff seine gesellschaftspolitische Relevanz erlang. Die Frage nach der Messung von Lebensqualität (und somit auch die Frage nach ihrer Definition) stellte sich - historisch betrachtet - häufig dann, wenn mithilfe des Konzeptes soziale Desiderata gelöst werden sollten. Aktuelle Diskussionen zur Bedeutung und zur Erfassung von Lebensqualität in der stationären Pflege weisen Ähnlichkeiten zu den bereits in der Vergangenheit geführten Debatten auf (Eppler 1974). Auch in der aktuellen Situation sind es Mängel, die mit- 
hilfe eines Monitorings von Pflege- und Lebensqualität behoben werden sollen (OECD und European Commission 2013). Befördert durch internationale Bemühungen um evidenzbasiertes Handeln und die Weiterentwicklung geeigneter Outcomes eines Healthy Ageing (WHO 2016), birgt eine solche Entwicklung Chancen als auch Risiken.

Die Chancen der geschilderten Entwicklung liegen in der (erstmaligen) Konzipierung von Lebensqualitätsansätzen, in denen die Perspektive der Bewohner und Bewohnerinnen von Pflegeeinrichtungen eine zentrale Rolle spielen soll. Die sowohl seitens der Politik als auch der Wissenschaft herausgestellte Notwendigkeit einer stärkeren Berücksichtigung von Lebensqualität in der stationären Pflege führt jedoch dazu, dass das Konzept zum Gegenstandsbereich der Gesundheits- und Pflegepolitik wird. Dessen Verwendung begünstigt dabei eine problemorientierte Indienstnahme anstatt einer theoriegeleiteten (Neu-)Entwicklung. Erste Auswirkungen einer politischen Verwertung des Begriffs zeigen sich an den Konturen seiner (Neu)Definition, z. B. an der Tendenz zur starken Akzentuierung eines negativen (= Schadensvermeidung) anstatt eines positiven (= Maximierung erwünschter Ergebnisse) Nutzens. Da ein theoretischer Konsensus fehlt, birgt dies die Gefahr, dass pflegebezogene Lebensqualität - auch unter der »Last « ungelöster methodischer Herausforderungen, z. B. bei der Konzipierung und Erfassung subjektiver Indikatoren, auf jene Aspekte der Qualität reduziert wird, die zuverlässig gemessen werden können. Die damit verbundene Entwicklung stellt Lebensqualität stärker in die Nähe medizinisch-klinischer Interventionen denn einer psychosozialen Versorgung mit Aspekten der Selbstbestimmung, Würde und sozialer Teilhabe. Dabei weisen viele - sowohl qualitative als auch quantitative - Studien darauf hin, dass gerade soziale Aspekte der Versorgung in stationären Pflegeeinrichtungen wichtig sind und sie häufig den Mittelpunkt individueller Lebensqualitätsvorstellungen von Bewohnern und Bewohnerinnen bilden. Nicht die (physische) Gesundheit, sondern Unabhängigkeit, Autonomie und Individualität (Lee et al. 2009) sowie autonome Lebensführung, soziale Kontakte, Versorgung, das Personal sowie die Lage und Ausstattung der Einrichtung sind wichtige Bereiche der Lebensqualität aus Sicht von Bewohnern und Bewohnerinnen (Josat et al. 2006). Angesichts der ebenfalls vorhandenen starken Plädoyers dafür, Lebensqualität als eine subjektive Konstruktion zu verstehen (Plummer und Molzahn 2009), kann die Lösung des Dilemmas nicht in der Reduktion des Konzeptes auf leicht erfassbare Daten oder die Adaption von Instrumenten zur Messung gesundheitsbezogener Lebensqualität gesucht werden. Vielmehr bedarf es der Entwicklung einer theoretischen (validen) Grundlage, in der Aspekte wie Selbstbestimmung, Würde und soziale Teilhabe in den Mittelpunkt gerückt werden und pflegebezogene Lebensqualität auf einem Begriff von Pflege beruht, der stärker in die Nähe von »social care« rückt. Ein solcher Rahmen würde die Idee der Person-Zentrierung nicht in Gefahr geraten lassen und langfristig dazu führen, dass zentrale Ziele eines Kulturwandels in der stationären Pflege - mehr Empowerment von Pflegebedürftigen sowie Anreize für Einrichtungen, Befragungsergebnisse tatsächlich zur Verbesserung der Lebensqualität einzusetzen (vgl. dazu Coulter et al. 2014) - ihren ursprünglich intendierten Stellenwert behalten. Die internationalen Beispiele zeigen mögliche Wege zur Realisierung solcher Konzepte, führen jedoch zugleich zu der Erkenntnis, dass eine an der Lebensqualität Pflegebedürftiger orientierte Weiterentwicklung der Qualität in der Pflege ein langfristiges Vorhaben darstellt, das einer sorgfältigen, die zentralen Ziele des Vorhabens reflektierenden Begleitforschung bedarf. 
Open Access Dieses Kapitel wird unter der Creative Commons Namensnennung 4.0 International Lizenz (http://creativecommons. org/licenses/by/4.0/deed.de) veröffentlicht, welche die Nutzung, Vervielfältigung, Bearbeitung, Verbreitung und Wiedergabe in jeglichem Medium und Format erlaubt, sofern Sie den/die ursprünglichen Autor(en) und die Quelle ordnungsgemäß nennen, einen Link zur Creative Commons Lizenz beifügen und angeben, ob Änderungen vorgenommen wurden.

Die in diesem Kapitel enthaltenen Bilder und sonstiges Drittmaterial unterliegen ebenfalls der genannten Creative Commons Lizenz, sofern sich aus der Abbildungslegende nichts anderes ergibt. Sofern das betreffende Material nicht unter der genannten Creative Commons Lizenz steht und die betreffende Handlung nicht nach gesetzlichen Vorschriften erlaubt ist, ist für die oben aufgeführten Weiterverwendungen des Materials die Einwilligung des jeweiligen Rechteinhabers einzuholen.

\section{Literatur}

Bishop M (2005) Quality of life and psychosocial adaptation to chronic illness and acquired disability: A conceptual and theoretical synthesis. Journal of Rehabilitation 71(2):5-13

Boggatz T (2016) Quality of life in old age - a concept analysis. International Journal of Older People Nursing 11(1):55-69

Coast J, Flynn TN, Natarajan L, Sproston K, Lewis J, Louviere JJ, Peters TJ (2008) Valuing the ICECAP capability index for older people. Social Science and Medicine 67(5):874-882

Cohen SR, Mount MM, Tomas JJN, Mount LF (1996) Existential well-being is an important determinant of quality of life. Cancer 77(3):576-586

Colombo F, Murakami Y (2013) Assessment and recommendations. In: OECD/European Commission. A good life in old age? Monitoring and improving quality in long-term care. OECD Publishing, Paris, S 15-33

Coulter A, Locock L, Ziebland S, Calabrese J (2014) Collecting data on patient experience is not enough: they must be used to improve care. BMJ 348: g2225

Damman OC (2010) Public reporting about healthcare users' experiences: the Consumer Quality Index. NIVEL, Utrecht

Damman OC, Stubbe JH, Hendriks M, Arah OA, Spreeuwenberg P, Delnoij DM, Groenewegen PP (2009) Using multilevel modeling to assess casemix adjusters in consumer experience surveys in health care. Medical Care 47(4):496-503

Delnoij DMJ, Rademakers JDJM, Groenewegen PP (2010) The Dutch Consumer Quality Index: an example of stakeholder involvement in indicator development. BMC Health Services Research10:88

Department of Health (2014) The Adult Social Care Outcomes Framework 2015/16. Department of Health, London

Department of Health (2017) The Adult Social Care Outcomes Framework 2016/2017. Handbook of Definitions. Department of Health, London www.gov.uk./government/ uploads/system/uploads/attachment_data/file/629812/ ASCOF_handbook_definitions.pdf. Zugegriffen: 15. November 2017

Eppler E (1974) Maßstäbe für eine humane Gesellschaft: Lebensstandard oder Lebensqualität? Kohlhammer, Stuttgart

Ferrans CE, Zerwic JJ, Wilbur JE, Larson JL (2005) Conceptual model of health-related quality of life. Journal of Nursing Scholarship 37(4):336-342
Ferrans CE (1990) Quality of life: Conceptual issues. Seminars in Oncology Nursing 6(4):248-254

Ferrans CE, Powers MJ (1985) Quality of Life Index: Development and psychometric properties. Advances in Nursing Science 8(1):15-24

Forder J, Netten A, Caiels J, Smith J, Malley J (2007) Measuring Outcomes in Social Care: Conceptual Development and Empirical Design. Quality Measurement Framework Project PSSRU Interim Report. PSSRU Discussion Paper 2422

Forder J, Malley J, Rand S, Vadean F, Jones K, Netten A (2016) Identifying the impact of adult social care: Interpreting outcome data for use in the Adult Social Care Outcomes Framework. PSSRU-Discussion Paper 2892

Foreman MD, Kleinpell R (1990) Assessing the quality of life of elderly persons. Seminars in Oncology Nursing 6(4): 92-297

Glatzer W, Zapf W (Hrsg) (1984) Lebensqualität in der Bundesrepublik. Objektive Lebensbedingungen und subjektives Wohlbefinden. Campus-Verlag, Frankfurt a. M.

Görres S, Rothgang H, Fünfstück M, Schmidt S, Seibert K, Siltmann S, Meyer J, Bendig J, Brannath W, Hasseler M, Buchner B (2017) Modellhafte Pilotierung von Indikatoren in der stationären Pflege (MoPIP). Abschlussbericht zum Forschungsprojekt. UBC-Zentrum für Alterns-und Pflegeforschung und UBC- Zentrum für Sozialpolitik an der Universität Bremen. www.gkv-spitzenverband.de/media/ dokumente/pflegeversicherung/qualitaet_in_der_pflege/ indikatoren/20170320_Erganzt_Abschlussbericht_MoPIP_Universitat_Bremen.pdf. Zugegriffen: 29. September 2017

Haas BK (1999a) A multidisciplinary concept analysis of quality of life. Western Journal of Nursing Research 21(6):728-742

Haas BA (1999b) Clarification and integration of similar quality of life concepts. Journal of Nursing Scholarship 31(3):215-220

Halvorsrud L, Kalfoss M (2007) The conceptualization and measurement of quality of life in older adults: a review of empirical studies published during 1994-2006. European Journal of Ageing 4:229-246

Herdman TH, Kamitsuru S(2017) Nursing Diagnoses: Definitions and Classification 2018-2020. 11. Auflage. Thieme, Stuttgart 
Herold-Majumdar A, Behrens J (2012) Lebensqualität im Fokus des Qualitätsaudits in der Langzeitpflege. Der Lebensqualitäts-Index (LQ-Index) - ein »Zauberstab« mit begrenzter Wirkung. Gesundheitswesen 74(12):806-811

Higgs P, Hyde M, Wiggin R, Blane D (2003) Researching Quality of Life in Early Old Age: The Importance of the Sociological Dimension. Social Policy and Administration 37(3):239-252

International Council of Nurses (2017) International Classification for Nursing Practice. Deutsche Übersetzung. www.icn.ch/images/stories/documents/pillars/Practice/ icnp/translations/icnp-German_translation.pdf. Zugegriffen: 25. September 2017

Josat S, Schubert H-J, Schnell MW, Köck C (2006) Qualitätskriterien, die Altenheimbewohnern und Angehörigen wichtig sind. Pflege 19(2):79-87

Kane RL (2015) A new long-term care manifesto. The Gerontologist 55(2):296-301

Kane RL, Kane RA (2015) The long view of long-term care: Our personal take on progress, pitfalls, and possibilities. Journal of the American Geriatrics Society 63(11):24002406

Kane RA, Kling KC, Bershadsky B, Kane RL, Giles K, Degenholtz HB, Liu J, Cutler LJ (2003) Quality of life measures for nursing home residents. Journal of Gerontology: Series A 58(3):M240-M248

Kelley-Gillespie N (2012) A secondary analysis of perceptions of quality of life of older adults residing in a nursing home and assisted setting using an integrated conceptual model of measurement. Applied Research Quality Life 7(2):137-154

Lawton MP (1991) A multidimensional view of quality of life in frail elders. In Birren JE, Lubben JE, Rowe JC, Deutchman DE. The Concept and Measurement of Quality of Life in the Frail Elderly. Academic Press, San Diego, S 3-27

Lee DTF, Yu DSF, Kwong ANL (2009) Quality of life of older people in residential care homes: a literature review. Journal of Nursing and Healthcare of Chronic Illness 1(2):116-125

Love K (2011) The Affordable Care Act: positive impact for quality improvement. Geriatric Nursing 32(4):282-284

Makai P (2012) Capabilities and quality of life in Dutch psychogeriatric nursing homes: An exploratory study using a proxy version of the ICECAP-O. Quality of Life Research 21(5):801-812

Malley JN, Towers A-M, Netten AP, Brazier JE, Forder JE, Flynn T (2012) An assessment of the construct validity of the ASCOT measure of social care-related quality of life with older people. Health and Quality of Life Outcomes, 10:21

McGee HM, O'Boyle CA, Hickey A, O'Malley K, Joyce CRB (1991) Assessing the quality of life of the individual: The SEIQoL with a healthy and a gastroenterology unit population. Psychological Medicine 21(3):749-759

Meyer J, Owen T (2008) Calling for an international dialogue on quality of life in care homes. International Journal of Older People Nursing 3(4)291-294
NCHRDF (2006) My home life. Quality of life in care homes. Help the Aged, London

Netten A, Beadle-Brown J, Trukeschitz B, Towers A-M, Welch E, Forder J, Smith J, Alden E (2010) Measuring the outcomes of care homes: Final Report. PSSRU Discussion Paper $2696 / 2$

Netten A, Beadle-Brown J, Caiels J, Forder J, Malley J, Smith N, Towers A-M, Trukeschitz B, Welch E, Windle K (2011) Adult Social Care Outcomes Toolkit. Main Guidance v2.1. PSSRU Discussion Paper 2716/3

Noll H-H (2000) Konzepte der Wohlfahrtsentwicklung: Lebensqualität und »neue« Wohlfahrtskonzepte. No P 00-505, Papers, Research Network Project »Work and Ecology«. Social Science Research Center (WZB), Berlin

OECD, European Commission (2013) A Good Life in Old Age? Monitoring and Improving Quality in Long-Term Care. OECD Health Policy Studies. OECD Publishing, Paris

Oswald F, Wahl H-W, Antfang P, Heusel Ch, Maurer A, Schmidt H (2014) Lebensqualität in der stationären Altenpflege mit INSEL. Konzeption, praxisnahe Erfassung, Befunde und sozialpolitische Implikationen. LIT, Münster

Padilla GV, Grant MM (1985) Quality of life as a cancer nursing outcome variable. Advances in Nursing Science 8(1):45-60

Pinto S, Fumincelli L, Mazzo A, Caldeira S, Carlos Martins J (2017) Comfort, well-being and quality of life: Discussion of the differences and similarities among the concepts. Porto Biomedical Journal 2(1):6-12

Plummer M, Molzahn AE (2009) Quality of life in contemporary nursing theory: A concept analysis. Nursing Science Quarterly 22(2):134-140

Pringle D, Doran DM (2003) Patient Outcomes as an Accoutability. In: Doran DM. Nursing Sensitive Outcomes - State of the Science. Jones and Bartlett Publishers, Mississauga, S 1-26

Rand S, Caiels J, Collins G, Forder J (2017) Developing a proxy version of the Adult social care outcome toolkit (ASCOT). Health and Quality of Life Outcomes 15:108

Roop JC, Payne JK, Vallerand AH (2012) Theories and conceptual models to guide quality-of-life research. In: King CR, Hinds PS. Quality of Life from Nursing and Patient Perspectives. Jones \& Bartlett Learning, Sudbury, USA, S 45-57

Ryff CD, Keyes CLM (1995) The structure of psychological well-being revisited. Journal of Personality and Social Psychology 69(4): 719-727

Schenk L, Meyer R, Behr A, Kuhlmey A, Holzhausen M (2013) Quality of life in nursing homes: results of a qualitative resident survey. Quality of Life Research 22(10):2929-2938

Silvestre JH, Bowers BJ, Gaard S (2015) Improving the quality of long-term care. Journal of Nursing Regulation 6(2):52-56

Sixma HJ, van Campen C, Kerssens JJ, Peters L (2000) Quality of care from the perspective of elderly people: the QUOTEElderly instrument. Age and Ageing 29(2):173-178

Steering Committee Responsible Care. Quality Framework Responsible Care (2007). www.zichtbarezorg.nl/mailings/ FILES/htmlcontent/VV\&T/Kwaliteitskader\%20Verantwoorde\%20Zorg\%20\%28english\%29.pdf. Zugegriffen: 23. April 2015 
Triemstra M, Winters SW, Kool RB, Wiegers TA (2010) Measuring client experiences in long-term care in the Netherlands: a pilot study with the Consumer Quality Index Long-term Care. BMC Health Serv Res 10: 95

Turnpenny A, Caiels J, Crowther T, Richardson L, Whelton B, Beadle-Brown J, Forder J, Apps J, Rand S (2015) Developing an Easy Read version of the Adult Social Care Outcomes Toolkit (ASCOT). PSSRU-Discussion Paper 2891

Vaarama M, Pieper R (2013) Quality of life and quality of care: an integrated model. In: Michalos A. Encyclopedia of Quality of Life and Well-Being Research. Springer, Dordrecht, S 5269-5276

Van Leeuwen KM, Bosmans JE, Jansen APD, Rand SE, Towers A-M, Smith N, Razik K, Trukeschitz B, van Tulder MW, van der Horst HE, Ostelo RW (2015). Dutch translation and cross-cultural validation of the Adult Social Care Outcomes Toolkit (ASCOT). Health and Quality of Life Outcomes 13:56

Winters S, Strating MH, Klazinga NS, Kool RB, Huijsman R (2010) Determining the interviewer effect on CQ Index outcomes: a multilevel approach. BMC Medical Research Methodology 10:75

Wingenfeld K, Kleina T, Franz S, Engels D, Mehlan S, Engels H (2011) Entwicklung und Erprobung von Instrumenten zur Beurteilung der Ergebnisqualität in der stationären Altenhilfe. Bericht im Auftrag des Bundesministeriums für Gesundheit und des Bundesministeriums für Familie, Senioren, Frauen und Jugend. Institut für Pflegewissenschaft an der Universität Bielefeld \& Institut für Sozialforschung und Gesellschaftspolitik, Köln

World Health Organization (2016) Multisectoral action for a life course approach to healthy ageing: draft global strategy and plan of action on ageing and health. SixtyNinth World Health Assembly. www.apps.who.int/iris/ handle/10665/252671. Zugegriffen: 15. November 2017

Zimmerman DR, Bowers BJ (2001) Integrating satisfaction surveys and other sources of information on quality of long-term care. In: Cohen-Mansfield J, Ejaz FK, Werner P. Satisfaction Surveys in Long-Term Care. Springer, New York, S 224-243

Zimmerman S, Shier V, Saliba D (2014) Transforming nursing home culture: Evidence for practice and policy. The Gerontologist 54 (Suppl. 1):S1-S5

Zubritsky C, Abbott KM, Hirschman KB, Bowles KH, Foust JB, Naylor MD (2013) Health-related Quality of Life: Expanding a Conceptual Framework to Include Older Adults Who Receive Long-term Services and Supports. The Gerontologist 53(2):205-210

Zuidgeest M, Delnoij DMJ, Luijkx KG, de Boer D, Westert GP (2012) Patients' experiences of the quality of long-term care among the elderly: comparing scores over time. BMC Health Services Research 12:26 